Theor et i cal anal ysi s of mode-compet i t i on noi se i $n$ modul at ed I aser di odes and its i nf I uence on the noi se performance of fibre li nks

\begin{tabular}{|l|l|}
\hline 著者 & Ahned Moust af a, Yanda M nor u \\
\hline $\begin{array}{l}\text { j our nal or } \\
\text { publ i cat i on t i t l e }\end{array}$ & Journal of Physi cs D: Appl i ed Physi cs \\
\hline vol une & 45 \\
\hline nunber & 40 \\
\hline page r ange & 405102 \\
\hline year & 2012- 10 - 01 \\
\hline URL & ht t p: //hdl . handl e. net /2297/32820 \\
\hline
\end{tabular}




\title{
Theoretical Analysis of Mode-Competition Noise in Modulated Laser Diodes and its Influence on Noise Performance of Fiber Links
}

\author{
Moustafa Ahmed ${ }^{1}$ and Minoru Yamda ${ }^{3}$ \\ ${ }^{1}$ Department of Physics, Faculty of Science, King Abdulazaiz University, Jeddah, Saudi \\ Arabia. \\ ${ }^{2}$ Division of Electrical Engineering and Computer Science, Graduate School of Natural \\ Science and Technology, Kanazawa University, Kakuma-machi, Kanazawa, Japan. \\ Email: mostafa.hafez@science.miniauniv.edu.eg
}

\begin{abstract}
We analyze the mode competition (MC) phenomenon in sinusoidally modulated laser diodes and the associated noise. Contribution of MC noise to the noise performance of directly modulated fiber links is evaluated in terms of the noise factor (NF) of the link. MC is analyzed through intensive simulation of laser dynamics and noise basing on a multimode rate equation model that takes into account both symmetric and asymmetric suppressions of the cross-modal gain. Variations of the MC relative intensity noise (RIN) of both the total output and the oscillating modes with modulation conditions are investigated. The obtained results showed that the modulated signal has six distinct waveforms depending on the modulation conditions. The modulated laser oscillates in single mode when the signal is continuous, and converts into multimode when the signal is pulsing. Contribution of MC noise to NF of the link increases with the increase in the modulation depth and with the decrease in the modulation frequency, except when the signal has period doubling. This contribution is negligible under high modulation frequencies when the laser signal is continuous and uniform, and is most enhanced $(\sim 53 \mathrm{~dB})$ under low-modulation frequencies when the signal is pulsing and superposed with non-uniform relaxation oscillations.
\end{abstract}

Keywords: Fiber link, mode competition, semiconductor laser, sinusoidal modulation PACS: $42.55 . \mathrm{Px}, 42.60 . \mathrm{Fc}, 42.60 . \mathrm{Mi}, 42.81 . U v$

\section{Introduction}

The ability to achieve single-mode oscillation with low noise and narrow bandwidth has made the in semiconductor laser attractive for use as a light source in several applications. The applications include fiber optic links, which are increasingly used for the distribution of radio frequency signals 
in mobile communication networks and WLANs. ${ }^{1}$ Primary factors affecting design of these RF fiber link include the overall cost of the components, linearity and noise [1]. Cost-effective RF fiber links apply the technology of direct modulation onto laser diodes [2].

Semiconductor lasers exhibit nonlinear phenomena that induce nonlinear forms of the modulated laser signal with harmonic distortions and enhanced noise levels [3]. These undesired effects limit the free dynamic range of both the laser and the fiber link, and degrades the noise performance of the link [3]. One of the most important sources of laser nonlinearities is the competition phenomenon among the oscillating modes [4]. In Fabry-Perot lasers, longitudinal MC is caused by both the self- and the spectrally symmetric and asymmetric cross-suppressions of modal gain, and is instantaneously modulated by the intrinsic fluctuations of the mode intensities [5]. Although MC may cause single-mode oscillation with very large values of the side mode suppression ratio (SMSR), the asymmetric suppression of modal gain may induce multi-stable operation of the laser [5]. In the latter case, the intrinsic noise of the laser causes mode hopping among the oscillating modes, which enhances the intensity fluctuations in the oscillating modes as well as in the total output [5-7]. A more serious concern with these modal dynamics is the manifestation of mode-partition noise due to fiber dispersion, a subject studied previously in considerable details [8]. This MC noise does not usually extend beyond a few gigahertz and is generally but erroneously not considered harmful to high frequency microwave systems. However, it has been shown that in a directly modulated laser diode, the low frequency noise can be translated to the neighborhood of the modulation carrier [9], which is a source of signal-induced noise at high frequencies. Although there are several reports on $\mathrm{MC}$ noise, its individual contribution to the overall noise of fiber links has not been evaluated.

Intensity modulation modulates the injected carriers in the active layer, which may stabilize or violate the modal dynamics depending on the modulation depth as well as the modulation frequency and its value compared with the hopping frequency in hopping multimode lasers. It was observed in experiments that the modulated signal of the laser has nonlinear forms with different degrees of signal distortion and noise levels in both regimes of low and high modulation frequencies $[10,11]$. Explanation of such effects in modulated laser diodes that support more than one mode 
oscillations and in the deploying fiber links was given phenomenologically in terms of the laser nonlinearity and mode competition [9]. Trustworthy analyses, however, of these frequency domain properties should correlate them with the time-domain characteristics of competition among the oscillating modes.

Recently, Ahmed et al. [12,13] has introduced comprehensive analyses of the modulation performance of semiconductor lasers and the associated noise basing on a large-signal model of single-mode oscillation. Such large-signal analyses were applied also to control optical feedback noise by means of sinusoidal modulation [15]. The modulated laser signal was found to be either continuous or pulsing; each has period doubling or is superposed by relaxation oscillations depending on the modulation conditions. Similar experimental results were also reported $[9,10]$. The relative intensity noise was found to be enhanced in both regimes of low and high modulation frequencies when the signal has irregular pulses or period doubling [12]. Therefore analyses of MC dynamics and noise under modulation are essential to evaluate the modulation performance of the laser as well as the noise performance of fiber links deploying laser diodes oscillating multimode.

In this paper, the large-signal model in Refs. $[12,13]$ of direct modulation is extended to the case of multimode oscillation. The present large-signal analysis treats the MC phenomenon basing on a multimode rate equation model that takes into account both self- and cross suppressions of modal gain. The intrinsic noise of semiconductor lasers is taken into account by means of Langevin noise sources in the rate equations of all modes as well as in the rate equation of the injected carriers. MC dynamics are then monitored as the time-domain characteristics of the modulated signal of the individual modes, while the frequency domain characteristics determine the frequency spectrum of RIN of the modulated signal. One-to-one correspondence is then traced between MC noise and the time-varying signal over wide ranges of the modulation conditions. Finally we evaluate contribution of MC RIN to the overall noise performance of a directly modulated fiber link employing a single mode fiber and a PIN photodiode. The link noise is evaluated in terms of NF which measures degradation of the signal-to-noise ratio [14]. These calculations take into account the laser noise and the shot and thermal noises of the receiver. The contribution of MC RIN to NF is evaluated by comparing the obtained NF results with those obtained when neglecting the laser 
noise. Comparison is also given for the results on MC noise under conditions of nearly single-mode and mode hopping oscillations.

The obtained results show that the modulated laser waveform is continuous periodic signal (CPS), continuous periodic signal with relaxation oscillations (CPSRO), continuous periodic signal with period doubling (CPSPD), periodic pulse (PP), periodic pulse with relaxation oscillations (PPRO) or periodic pulse with period doubling (PPPD). This classification is independent of the modal oscillation, whether it is single mode or multimode. The laser is proved to oscillate in single mode when the signal is continuous, whereas the pulsing signals are characterized by multimode oscillation. Sinusoidal modulation is shown to stabilize mode hopping instabilities and suppress the associated noise nearly to the levels of nearly single-mode oscillation. The total MC RIN increases in general with the increase in the modulation depth. The contribution of MC RIN to NF of the fiber link is most enhanced $(\sim 53 \mathrm{~dB})$ under the PPRO type in the regime of low-frequency modulation. Such contribution is lowest under weak and high-frequency modulation where the laser supports the CPS type.

The paper is organized as follows. In the following section, the simulation model of MC dynamics and noise under modulation is introduced. A simulation model of NF of a directly modulated fiber link is also given. Section 3 introduces the calculation procedures of MC in both the time and frequency domains. Section 4 presents the results of large-signal computer simulation of MC dynamics and noise as well as its contribution to NF of the fiber link. General conclusions of the present work appear in the last section.

\section{Theoretical model of analysis}

\subsection{MC dynamics and noise of semiconductor lasers}

The present analysis of MC dynamics and noise of semiconductor lasers under modulation is based on the following multimode rate equation model of the injected carrier number $N(t)$ and modal photon number $S_{p}(t)$ with $p=0, \pm 1, \pm 2, \ldots$ : 


$$
\begin{gathered}
\frac{d N}{d t}=\frac{1}{e} I(t)-\sum_{p} A_{P} S_{p}-\frac{N}{\tau_{s}}+F_{N}(t) \\
\frac{d S_{p}}{d t}=\left(G-G_{p}\right) S_{p}+C_{p} \frac{N}{\tau_{s}}+F_{S_{p}}(t)
\end{gathered}
$$

where $G_{p}$ is the gain of mode $p$ whose wavelength is $\lambda_{p} . G_{p}$ is defined such that the suppression by mode $p$ itself and both the symmetric gain suppression (SGS) and asymmetric gain suppression (AGS) by other modes $q \neq p$ are taken into account [5,6],

$$
G_{p}=A_{p}-S_{p}-\sum_{q \neq p}\left[D_{p(q)}+H_{p(q)}\right\rfloor S_{q}
$$

$A_{p}$ is the linear gain, $B$ is the coefficient of self-suppression, and $D_{p(q)}$ and $H_{p(q)}$ are coefficients of SGS and AGS, respectively. These coefficients are given by $[5,6]$ :

$$
\begin{aligned}
A_{p} & =\frac{a \xi}{V}\left[N-N_{g}-b V\left(\lambda_{p}-\lambda_{\text {peak }}\right)^{2}\right] \\
B & =\frac{9}{2} \frac{\pi c}{\varepsilon_{0} n_{D}^{2} \hbar \lambda_{0}}\left(\frac{\xi \tau_{i n}}{V}\right)^{2} a\left|R_{c v}\right|^{2}\left(N-N_{s}\right) \\
D_{p(q)} & =\frac{4}{3} \frac{B}{\left.2 \pi c \tau_{\text {in }} / \lambda_{p}^{2}\right)\left(\lambda_{p}-\lambda_{q}\right)^{2}+1} \\
H_{p(q)} & =\frac{3}{8 \pi}\left(\frac{a \xi}{V}\right)^{2}\left(N-N_{g}\right) \frac{\alpha \lambda_{p}^{2}}{\lambda_{q}-\lambda_{p}}
\end{aligned}
$$

In Eq. (4), $a$ is the differential gain, $\xi$ is the field confinement factor, $\lambda_{\text {peak }}$ is the wavelength at the peak of the linear gain spectrum $A_{p}$ whose width is $b$, and $N_{g}$ is the electron number at transparency. In the nonlinear gain equations (5)-(7), $\tau_{i n}$ is the intraband relaxation time, $R_{c v}$ is the dipole moment, $N_{s}$ is an electron number characterizing $B$, and $\alpha$ is the linewidth enhancement factor. Remaining parameters are: $I$ is the injection current, $n_{D}$ is the refractive index of the laser cavity whose volume is $V$ and length is $L_{D}, e$ is the electron charge, $\tau_{s}$ is the electron lifetime by spontaneous emission, and $\bar{N}$ is the time-average value of $N(t)$. Inclusion of the spontaneous emission into the lasing mode is described in terms of the spontaneous emission factor $C_{p}$, which is given as [5] 


$$
C_{p}=\frac{a \xi \tau_{s} / V}{1+\left(2 \frac{\lambda_{p}-\lambda_{\text {peak }}}{\delta \lambda}\right)^{2}}
$$

The central mode $p=0$ with wavelength $\lambda_{0}$ is assumed to lie at the peak of the gain spectrum, and the wavelength of the other modes is defined as:

$$
\lambda_{p}=\lambda_{0}+p \Delta \lambda=0, \pm 1, \pm 2, \pm 3, \ldots
$$

where $\Delta \lambda=\lambda_{0}^{2} / n_{D} L_{D}$ is the modal wavelength separation. The mode index $p$ is positive for modes lying on the long wavelength side of the central mode, i.e., $\lambda_{p}>\lambda_{0}$, and is negative for modes with shorter wavelengths, $\lambda_{p}<\lambda_{0}$. Equation (7) indicates that the AGS coefficient $H_{p(q)}$ is inversely proportional to $\lambda_{q}-\lambda_{p}$. Therefore, AGS of mode $p$ works to suppress gain of modes $q \neq p$ with shorter wavelength but enhance gain of the modes with longer wavelengths. Such influence is pronounced in lasers made from InGaAsP materials due to the large values of the $\alpha$-factor [16], and with rather long cavities due to the rather short values of $\Delta \lambda$.

The sinusoidal modulation is included in the rate equations by representing the timevarying current $I(t)$ in rate equation (1) of $N(t)$ by the following sinusoidal form

$$
I(t)=I_{b}\left\{1+m \cos \left(2 \pi f_{m} t\right)\right\}
$$

where $I_{b}$ is the bias current, $m$ is the modulation depth and $f_{m}$ is the modulation frequency.

The last terms $F_{N}(t)$ and $F_{S p}(t)$ in equations (1) and (2) are Langevin noise sources with zero mean values. They are added to the rate equations to account for intrinsic fluctuations of the laser associated with quantum transitions of electrons between the valence and conduction bands [17]. These noise sources are assumed to have Gaussian probability distributions and are $\delta$-correlated processes with the correlations [18] [5]

$$
\begin{aligned}
\left\langle F_{S_{p}}(t) F_{S_{q}}\left(t^{\prime}\right)\right\rangle & =V_{S_{p} S_{q}} \delta\left(t-t^{\prime}\right) \\
\left\langle F_{N}(t) F_{N}\left(t^{\prime}\right)\right\rangle & =V_{N N} \delta\left(t-t^{\prime}\right) \\
\left\langle F_{S_{p}}(t) F_{N}\left(t^{\prime}\right)\right\rangle & =V_{S_{p} N} \delta\left(t-t^{\prime}\right)
\end{aligned}
$$


where $\delta$ is the Dirac's delta-function. In the above equations, the property of orthogonality among modes is introduced in terms of the Kronecker delta $\delta_{p, q}$. The variances of the noise sources $V_{x y}$ (with $x$ and $y$ referring to any of $S_{p}$ or $N$ ) are determined as sums of variances of the fluctuating terms in the rate equations $[17,18]$.

The frequency content of the mode fluctuations is measured in terms of RIN, which is calculated from the fluctuations $\delta S(t)=S(t)-S_{b}$ in the total photon number $S(t)=\sum_{p} S_{p}(t)$, where $S_{b}$ is the bias value of $S(t)$. Over a finite time $T$, RIN is given as [17]

$$
R I N=\frac{1}{S_{b}^{2}}\left\{\frac{1}{T}\left|\int_{0}^{T} \delta S(t) e^{-j \Omega \tau} d \tau\right|^{2}\right\}
$$

where $f=\Omega / 2 \pi$ is the Fourier frequency. Similarly, RIN of the individual modes is defined in terms of the fluctuations of their photon number $S_{p}(t)$. The noise performance of the laser diode is evaluated also in terms of the average value of the RIN components at frequencies lower than 100MHz. This low-frequency RIN (LF-RIN) level is important to describe the noise associated with MC.

\subsection{Noise in directly modulated fiber links}

We assume an RF fiber link deploying a directly modulated laser diode whose modulated signal is transmitted over a lossy single-mode fiber. At the other end of the fiber, a PIN photodiode recovers the RF signal from the optical carrier. The fiber link is assumed so short that fiber dispersion is not considered. Two passive impedance-matching circuits are supposed to match the laser and PIN impedances to the RF source and load impedances, respectively [2]. The PIN photocurrent is related to the launched power $P$ from the laser through the fiber attenuation $\alpha_{\text {fiber }}$ and photodiode responsivity $R_{0}$ as

$$
I_{\mathrm{ph}}=R_{0} \alpha_{\text {fiber }} P
$$

The laser power $P$ is calculated from the average value $\bar{S}$ of the total photon number via the relationship [6] 


$$
P=\frac{h c^{2}}{2 n_{\mathrm{D}} L_{\mathrm{D}} \lambda_{\text {peak }}} \frac{\left(1-R_{f}\right) \ln \left(1 / R_{f} R_{b}\right)}{\left(1-\sqrt{\left.R_{f} R_{b}\right)}\left(1-\sqrt{\left.R_{f} R_{b}\right)}\right.\right.} \bar{S}
$$

The output electrical power through the load resistance $R_{L}$ of the PIN is given by [2]:

$$
P_{\text {out }, \text { electr }}=\frac{N_{d}^{2} I_{p h}^{2} R_{L}}{\left(R_{S}+N_{d}^{2} R_{L}\right)\left(2 \pi f_{m} C_{d}\right)^{2}+1}
$$

The input (source) electrical power $P_{S}$ to the link is given in terms of a matching resistance to the laser diode $R_{s}$ and the average value of the modulating current $\langle I\rangle$ as

$$
P_{\mathrm{S}}=2 R_{S}\langle I\rangle^{2}
$$

The link gain (loss) is an important performance criterion of the link, and is defined as the ratio of the power delivered to the load $P_{\text {out }, \text { electr }}$ to the power available from the source $P_{s}[1]$

$$
G_{\text {link }}=\frac{P_{\text {out }, \text { electr }}}{P_{\mathrm{S}}}
$$

In addition to the laser noise, the total noise of the link includes the thermal noise and shot noise in the PIN. NF of the link is related to the link gain $G_{\text {link }}$ through the expression [2]

$$
N F(d B)=10 \log _{10}\left(\frac{N_{\text {out, thermal }}+N_{\text {out, RIN }}+N_{\text {out, shot }}}{k T B G_{\text {Link }}}\right)
$$

where $N_{\text {out }}$ is the electrical power dissipation caused by the corresponding noise currents in the load resistance. These noises are given in terms of the bandwidth of the receiver $B$ as [1]

$$
\begin{aligned}
N_{\text {out, RIN }} & =\left\langle i_{R I N}^{2}\right\rangle R_{L}=R_{L} I_{p h}^{2} B R I N \\
N_{\text {out, shot }} & =\left\langle i_{\text {shot }}^{2}\right\rangle R_{L}=2 q R_{L} B\left(I_{p h}+I_{d}\right) \\
N_{\text {out, thermal }} & =\left\langle i_{\text {thermal }}^{2}\right\rangle R_{L}=4 k B T
\end{aligned}
$$

Definitions of other parameters appeared in the above equations are given in table 1.

\section{Procedures of Numerical Calculation}

3.1. Deterministic modulation dynamics of mode competition 
To gain insight into the MC phenomenon under modulation, the Langevin noise sources are dropped from the modal rate equations (1) and (2). The equations are then solved numerically using an integration step of $\Delta t=10 \mathrm{ps}$. A large number of 15 modes are counted in the calculations to ensure accuracy of analyzing MC. The laser is assumed to be biased above threshold to neglect contribution of the noisy spontaneous emission to the laser output. 850-nm AlGaAs laser diodes are assumed for the present calculations. Typical values of the parameters of this laser are listed in table 1.

The bias component $S_{b}$ of the total photon number $S(t)$ is evaluated via the equation

$$
S_{b}=\frac{I_{b}-I_{t h}}{e G_{t h}}
$$

where $I_{t h}$ is the threshold current. The corresponding relaxation oscillation frequency $f_{r}$ is determined approximately from the small-signal approach as [13]

$$
f_{r} \approx \frac{1}{2 \pi} \sqrt{\left(\frac{a \xi}{V}\right)\left[\frac{a \tau_{e}}{e V}\left(I_{b}-I_{g}\right)+B \frac{I_{b}-I_{t h}}{e G_{t h}}\right] \frac{I_{b}-I_{t h}}{e G_{t h}}}
$$

where $I_{g}=e N_{g} / \tau_{s}$ is the current at transparency.

The output spectrum of the laser is calculated by averaging the temporal modal photon numbers $S_{p}(t)$ and constructing a bar plotting of these average values $\overline{S_{p}}$ versus the mode index $p$. SMSR of the multimode oscillation is evaluated as the ratio of the average value of the dominant mode (DM) to that of the strongest side mode (SSM). In the present work, the condition of single mode oscillation is defined as SMSR $>50$.

The frequency content of the modulated laser signal is determined by the frequency spectrum of the fast Fourier transform (FFT) of the temporal trajectory of $S(t)$. The FFT power spectrum is calculated over a finite period $T$ as

$$
S_{f}=\frac{1}{T}\left|\int_{0}^{T} S(t) e^{-j \Omega \tau} d \tau\right|^{2}=\sqrt{\frac{\Delta t}{T}}|F F T(S(t))|
$$

\subsection{MC noise}


Rate equations (1) and (2) including the Langevin noise sources are integrated by the fourth-order Runge-Kutta method. At each integration instant $t_{i}$, the noise sources $F_{S p}\left(t_{i}\right)$ and $F_{N}\left(t_{i}\right)$ are generated using the following forms [5]

$$
\begin{aligned}
F_{S_{p}}\left(t_{i}\right) & =\sqrt{\frac{V_{S_{p} S_{q}}}{\Delta t} g_{S_{p}}} \\
F_{N}\left(t_{i}\right) & =\sqrt{\frac{V_{N N}\left(t_{i}\right)+\sum_{p} k_{p}\left(t_{i}\right) V_{S_{p} N}\left(t_{i}\right)}{\Delta t}} g_{N}-\underset{p}{-\sum_{p} k_{p}\left(t_{i}\right) F_{S_{p}}\left(t_{i-1}\right)}
\end{aligned}
$$

where the modal coefficient $k_{p}$ is calculated from the variances $V_{S p N}$ and $V_{S p S q}$ at time $t_{i}$ as

$$
k_{p}\left(t_{i}\right)=\frac{V_{S_{p} N}\left(t_{i}\right)}{V_{S_{p} S_{q}}\left(t_{i}\right)}
$$

The variances $V_{x y}$ (with $x$ and $y$ referring to each of the symbols $S_{p}$ or $N$ ) at time $t_{i}$ are evaluated from $S\left(t_{i}-1\right)$ and $N\left(t_{i}-1\right)$ at the preceding time $t_{i}-1$ by assuming quasi-steady states $\left(\mathrm{d} S_{p} / \mathrm{d} t \approx\right.$ $\mathrm{d} N / \mathrm{d} t \approx 0$ ) over the integration step $\Delta t=t_{i-1}-t_{i}$,

$$
\begin{aligned}
V_{S_{p} S_{p}}\left(t_{i}\right) & =2\left[\frac{a \xi}{V} S_{p}\left(t_{i-1}\right)+\frac{C_{p} \xi}{\tau_{s}}\right] N\left(t_{i-1}\right) \\
V_{N N}\left(t_{i}\right) & =2\left[\frac{1}{\tau_{s}}+\frac{a \xi}{V} \sum_{p} S_{p}\left(t_{i-1}\right)\right] N\left(t_{i-1}\right) \\
V_{S_{p} N}\left(t_{i}\right) & =-\frac{a \xi}{V}\left[N\left(t_{i-1}\right)-N_{g}\right] S\left(t_{i-1}\right)-C_{p} \frac{N\left(t_{i-1}\right)}{\tau_{s}}
\end{aligned}
$$

In Eqs. (13) and (14), $g_{s p}$ and $g_{N}$ are independent Gaussian random numbers with variances of unity and zero mean values. They are obtained at each integration step by applying the Box-Mueller approximation [19] to a set of uniformly distributed random numbers generated by the computer.

The noise content of the MC is evaluated in terms of the frequency spectrum of RIN of the oscillating modes and the total laser output. RIN of the total output is calculated from the fluctuations $\delta S\left(t_{i}\right)$ via Eq. (13) as 


$$
R I N=\frac{1}{{\overline{S_{b}}}^{2}} \frac{\Delta t^{2}}{T} \mid F F T\left[\left.\delta S\left(t_{i}\right)\right|^{2}\right.
$$

RIN of the individual modes are calculated in similar fashion from $\delta S_{p}\left(t_{i}\right)$.

\subsection{Noise in fiber link}

The noise performance of the designed RF fiber link is evaluated in terms of NF, which is calculated from Eq. (19) including MC RIN. The used values of the link parameters are listed in table 1.

\section{Results and Discussion}

\subsection{RIN of non-modulated laser}

Figure 1 characterizes the mode dynamics and noise when MC results in unstable mode hopping $\left(I=2.74 I_{t h}\right)$ and nearly single mode oscillation $\left(I=3.0 I_{t h}\right)$. Figures $1(\mathrm{a})$ and (b) plot the time variations

of the photon numbers of the most dominant modes normalized by the time-average value $\bar{S}$ of the total photon number. The corresponding output spectra and modal gain spectra are plotted in figures 1(c) and (d), while the frequency spectra of RIN are plotted in figures 1(e) and (f) for $I=2.74$ and 3.0I $I_{t h}$, respectively. Figure 1(a) shows that MC results in a transient random exchange of the mode intensity between the dominant modes $p=0$ and +1 , which is seen in the form of abrupt switching or hopping. In this case, $\mathrm{MC}$ induces a bistable state in the maser dynamics, and inclusion of the noise sources triggers random switching of modes $p=0$ and +1 between the bi-stable states signifying the hopping phenomenon [5,6,22]. Mode hopping is a famous phenomenon in laser dynamics and is observed in experiments [7,22]. The total power is mainly contained in the hopping modes as seen in the two-mode hopping output spectrum of figure 1(b). The corresponding SMSR is 1.7. On the other hand, figure 1 (b) indicates operation in single mode $p=+2$ with the time variation. The DM $p=+2$ varies almost independently of the other side modes, and the main mode 
intensity $S_{+2}(t)$ is close to the total intensity $S(t)$. The single-mode oscillation is indicated also in the output spectrum of figure 2(b), SMSR $=\bar{S}_{+2} / \bar{S}_{+3}=383$. The MC dynamics are controlled by AGS which results in the asymmetric gain spectra shown in figures 1(c) and (d). This AGS works to enhance the gain of modes on the long-wavelength side of mode $p=0$ and suppress the gain of modes on the shorter side [20,21]. As shown in figures 1(c) and (d), the single-mode oscillation corresponds to gain difference of 0.0049 between DM $\mathrm{p}=+2$ and SSM $p=+3$, which is reduced to 0.0035 between modes $p=+1$ and $p=0$ in the case of mode hopping.

The frequency spectra of RIN in figures 1(e) and (f) exhibit sharp peaks around the relaxation frequency $f_{r}$ of the laser. The observed increase in $f_{r}$ from figure $1(\mathrm{e})$ to (f) is due to the corresponding increase in the injection current $I$, which increases the rate of the relaxation oscillations [23]. The low-frequency components of RIN of the total output are higher under the mode hopping than under the single mode oscillation, which is due to the instabilities associated with mode hopping by MC. These results are in good fit with those obtained in theory and experiments [24]. Concerning modal noise, RIN of the hopping modes $p=+2$ and +3 in figure $1(\mathrm{e})$ drops smoothly from high levels $(-70.5$ and $-65.6 \mathrm{~dB} / \mathrm{Hz}$, respectively) and then turn to increase around $f_{r}$. Around $f=f_{r}$, RIN of the DM is almost coincident with that of the total output, while the levels of RIN of the SSM mode is lower. In the case of the nearly single-mode oscillation, figure 1(f) shows that the DM RIN decreases slowly in the low-frequency regime from a lower level (-78.8 $\mathrm{dB} / \mathrm{Hz}$ ), and so does the SSM $p=0$. Around $f_{r}$, the DM RIN reveals the resonance peak (coinciding with the RIN of the total output), while the SSM RIN drops rapidly to lower values. These characteristics of modal RIN are in good correspondence with those predicted in theory and measured in experiments [5-7,25-27]. 


\subsection{Modulation characteristics and harmonic distortion}

\subsubsection{Laser waveforms and modal oscillation under modulation}

Sinusoidal modulation of the semiconductor laser is found to generate six distinct forms of the output signal. In this subsection, we introduce deterministic characterization of these signal types. The characterization is given in terms of (a) the signal waveforms of the modes and the total output, (b) spectra of mode intensities and gain, and (c) FFT frequency spectrum of the laser output and the associated harmonic distortion.

Figure 2 characterizes the case CPS in which $S(t)$ varies continuously and periodically with the time variation as shown in figure 2(a). The figure corresponds to $f_{m}=f_{r}$ and $m=0.1$. This time variation becomes sinusoidal under very weak modulation $(m<0.05)$. This type of signals was observed in experiments by Henery et al. [11] and simulated by Ahmed and El-Lafi [12]. In this case, the laser output is mainly contained in mode $p=+2(\mathrm{SMSR}=230)$ as indicated in the output spectrum of figure 2(b). The mode gain spectrum indicates the highest gain of the DM $p=+2$. Therefore, the signal shown in figure 2(a) corresponds to both $S_{+2}(t)$ and the total photon number $S(t)$. The FFT power spectrum $S_{f}$ in figure 2(c) is characterized by a sharp peak at $f_{m}$ and a weak peak at $2 f_{m}$. Therefore, this type has $2^{\text {nd }}$ harmonic distortion

$$
2 \mathrm{HD}(\mathrm{dB})=20 \log _{10}\left(\frac{a_{2}}{a_{0}}\right)
$$

as weak as $-9 \mathrm{~dB}$, where $a_{0}$ and $a_{1}$ are the peaks of the fundamental and second harmonics. This weak harmonic distortion reflects the high degree of periodicity of the signal.

With the increase in the modulation depth $m$ in the region of low-frequency modulation, the gain decreases further under the threshold level $G_{t h}$ around the valleys of the modulating current signal $I(t)$. This effect results in the CPSRO type as given in the waveform of figure 3(a). The shown signal corresponds to $f_{m}=0.1 f_{r}$ with $m=0.5$, and was observed also in the experiments of Henery et al. [11] and predicted by Ahmed and El_Lafi in single-mode lasers [13]. In this case also, the laser output is mainly contained in mode $p=+2$ (SMSR=237) as indicated in the output spectrum of figure 3(b). Therefore, the signal shown in figure 3(a) corresponds to both the DM $p=+2$ and the 
total output. The FFT spectrum $S_{f}$ in figure 3(c) is also characterized by a sharp peak at $f_{m}$ and a weak peak at $2 f_{m}$. $2 \mathrm{HD}$ is -17.2 , which is little higher than the case of the CPS type

Under high modulation frequencies, the continuously varying laser intensity exhibits doubling, where the modal gain goes far below $G_{t h}$. Therefore, the effect of the turn-on delay is pronounced in the time variation of $S(t)$ in the form of two unequal peaks. This CPSPD type is characterized in figure 4 , which corresponds to $f_{m}=1.4 f_{r}$ and $m=1.3$. Figure 4 (a) shows that the total photon number $S(t)$ has two peaks of different peaks in every two successive periods. The separation of each two successive similar peaks is equal to $2 T_{m}=f_{m} / 2$. In this type, the laser still oscillates in single mode, $p=+2$, but with lower value of $S M S R=164$. This is illustrated in the output and gain spectra of figure 4(b). The irregularity of the CPSPD is indicated in the FFT frequency spectrum figure $4(\mathrm{c})$ in terms of the pronounced peaks at $f_{m}$ and $f_{m} / 2$ as well as the higher harmonics. Therefore this type has half harmonic distortion (HHD) of $-1.7 \mathrm{~dB}$, which is much higher than $2 \mathrm{HD}=-7 \mathrm{~dB}$.

Under rather strong modulation, the continuous variation of the laser Intensity converts to pulsation due to stronger drop of gain under $G_{t h}$ during the valleys of $I(t)$. The uniform time variation is in the form of PPs, which is characterized in figure 5. In this case, $f_{m}=f_{r}$ and $m=1.2$. Figure 5(a) shows that $S(t)$ exhibits uniform PPs. In this case, the drop of gain under threshold is $G$ $G_{t h} / G_{t h}=0.001$, which is smaller than the value of 0.0028 of the CPSPD type. When the pulse is too short, this type corresponds to the spike generation predicted by Lee et al. [28]. Contrary to the cases of continuous variation of $S(t)$, the laser output is contained in several modes, as shown by the time trajectories of the strongest modes $p=+1$ and +2 in figure 5(a). This multimode oscillation is indicated also in the multimode spectrum of figure 5(b) with SMSR=5.6. Figure 5(c) plots the FFT spectrum $S_{f}$, which has peaks at $f_{m}$ and the higher harmonics. In this case $2 \mathrm{HD}=-2.4 \mathrm{~dB}$ which is much higher than that of the CPS type.

Under low-modulation frequencies where the modulation period $T_{m}$ is long enough for the relaxation oscillations to appear, the periodic pulsation is superposed by relaxation oscillations when strong modulation enhances the turn-on effect. Figure 6(a) plots the time characteristics of this CPSRO type when $f_{m}=0.1 f_{r}$ and $m=1.2$. In this case the laser output and the modal gain 
spectrum are homogeneously distributed among the oscillating modes as shown in figure 6(b), and the output spectrum is of a typical multimode type. The FFT frequency spectrum of figure 6(c) indicates a pronounced peak at $f_{m}$ and smaller peaks at $2 f_{m}$ and $3 f_{m}$. The corresponding harmonic distortion is $2 \mathrm{HD}=-5.1 \mathrm{~dB}$, which is higher than that of the CPSRO type.

The period pulsation happens also to exhibit period doubling under strong modulation with high modulation frequencies. This PPPD type is characterized in figure 7 when $m=1.3$ and $f_{m}=f_{r}$. Figure 7(a) shows that the pulses of $S(t)$ have a pulse every two successive periods, that is, the peak separation is $2 T_{m}=f_{m} / 2$. Contrary to the CPSPD type, this type is characterized by spectral homogenous distribution of modal gain and typical multimode output as shown in figure 7(c). The period doubling dynamics were observed in experiments by Chusseau et al. [10] and Henery et al. [11] and predicted in theory $[13,15]$. Figure $7(\mathrm{c})$ reflects the harmonic distortions associated with this type of the modulated signal. The values of HHD and2HD are $-7 \mathrm{~dB}$ and $-2.9 \mathrm{~dB}$, respectively.

\subsubsection{Modulation index versus modulation frequency diagram of modulation dynamics and modal} oscillation

In the previous subsection, we investigated the possible waveforms of the modulated laser signal, and characterized these types in both the time and frequency domains. It is of practical interest to investigate the modulation conditions that correspond to both uniform laser output that have low levels of noise and non-uniform output in order to avoid signal distortion and noise enhancement. For such purposes, we simulated the laser dynamics over wide ranges of the modulation depth, $m=$ $0.01-1.5$, and modulation frequency, $f_{m}=0.05 f_{r}-f_{3 d B}$, where $f_{3 d B} \approx 1.6 f_{r}$ [19] is the modulation bandwidth frequency. The modulation type corresponding to each modulation condition $\left(m, f_{m}\right)$ was then decided as discussed above. The modulation parameters, $m$ and $f_{m}$, corresponding to each of the investigated modulation types are mapped in the ( $m$ versus $f_{m}$ ) diagram of figure $8(\mathrm{a})$. Therefore, each domain in the diagram encloses modulation points ( $m$ versus $f_{m}$ ) that reveal the modulated laser output of designated type. Figure 8(a) includes also a contour plotting of SMSR as a function of $m$ and $f_{m}$, which points out the modal oscillation of the laser over the entire ranges of modulation conditions. 
The figure shows that at each value of $f_{m}$, the CPS type dominates the range of low values of $m$. The range of $m$ in the region of the CPS type increases with the increase in $f_{m}$. At a given value of $m$ within this CPS region, the signal amplitude increases with the increase in $f_{m}$ revealing a peak around the relaxation frequency $f_{r}$ and then decreases with further increase in $f_{m}$. This behavior fits the famous small-signal modulation response [30]. Up to $f_{m}=0.4 f_{r}$, the increase in $m$ converts the CPS type into the CPSRO type and then to the PPRO type. Within the operating region of the PPRO type, the degree of this non-uniformity increases with the increase in $m$. At rather high values of $0.4<f_{m} / f_{m}<1$, strengthening the modulation converts the CPS type directly into the discontinuous PP type, which agrees with the predictions of Kao and Lin [31]. Therefore, it can be decided that this range of $f_{m}$ corresponds to uniform and periodic laser output; it is either CPS or PP regardless of the relevant range of $m$. At a given value of $f_{m}$, the pulse width decreases with the increase in $m$. At higher values of $f_{m}$, when $m$ increases beyond 1.2, the period doubling effect appears; it starts with the CPSPD type and then converts into the PPPD type. Other forms of the PPPD include one pulse (with equal or little unequal peaks) every two successive periods. The degree of this non-uniformity increases with the increase in $m$.

The contour plot of SMSR in figure 8(a) shows that the laser almost operates in single mode, $p=+2$, when the signal exhibits continuous variation with time, whether the signal has the CPS, CPSRO or CPSPD type. However, near the boundaries between the continuous variation and pulsation, the value of SMSR decreases and the operation converts to multimode. Within the operating regions of pulsing outputs, SMSR decreases remarkably with the increase in $m$, which is associated with an increase in the width of the spectrum. These results are in good agreement with the experimental observations by Lau et al. [32] that under strong modulation, the single-mode lasing spectrum breaks into multimode oscillation and the envelope width of the spectrum increases rapidly with the increase in the modulation depth.

Figure $8(\mathrm{~b})$ plots the corresponding ( $m$ vs. $f_{m}$ ) diagram when the laser is assumed to oscillate primarily in single mode. In this case, the system of rate equations (1) of modal photon numbers is reduced to single equation of the mode oscillating at the peak, $\lambda_{\text {peak }}$, of the linear gain. Compared with the diagram of figure $8(a)$, minor variations occur at the boundaries of the operating 
regions of the signal types. Therefore, this comparison indicates an interesting result that the present characterization of the modulated waveform of the total laser output is independent on the modal oscillation of the laser. To assure this result, we also constructed a similar diagram when the laser operation reveals two-mode oscillation, and we obtained minor changes in the diagram.

\subsection{MC noise associated with modulation}

In this section, the spectral characteristics of MC RIN under sinusoidal modulation are investigated. Variation of the LF-RIN level with the modulation parameters is also discussed. The noise properties of each investigated type of the modulated waveform are compared with those of the non-modulated laser. Figures 9(a)-(f) plot typical frequency spectra of MC RIN in the investigated six types of the modulated signal: CPS, CPSRO, CPSPD, PP, PPRO and PPPD, respectively. The RIN spectrum of the non-modulated laser is also plotted in the figures for comparison. The operating conditions are the same as those of the characterizing figures $2-7$, respectively.

Figure 9(a) plots a typical frequency spectrum of RIN of the CPS type. This RIN spectrum has almost the same levels of that of the non-modulated laser, except that LF-RIN is almost $7 \mathrm{~dB} / \mathrm{Hz}$ higher and the spectrum has sharp peaks at $f_{m}$ and the higher harmonics. This small difference of the LF-RIN levels originates from the asymmetry of the CPS. Figure 9(b) characterizes RIN of the CPSRO type, showing that the RIN spectrum is about $20 \mathrm{~dB} / \mathrm{Hz}$ higher than that of the CPS type with sharp characteristic peaks at $f_{m}$ and the higher harmonics. LF-RIN is flat with a level of $\sim-132 \mathrm{~dB} / \mathrm{Hz}$ which is higher than those of both the non-modulated laser and CPS type. The last investigated type with a continuous time variation is the CPSPD type in which the signal has one peak in every two successive periods. This type is characterized in figure 9(c), which shows that the low-frequency components are also flat with LF-RIN $\sim-140 \mathrm{~dB} / \mathrm{Hz}$. This noise level is still higher than that of either the CPS type or the non-modulated laser. The characteristic RIN spectrum of the PP type is given in figure 9(d), which shows that the RIN spectrum is characterized by sharp peaks at $f_{m}$ and its multiples. The figure shows also that the RIN spectrum is almost $30 \mathrm{~dB} / \mathrm{Hz}$ higher than that of the non-modulated laser. The noise content of the PPRO type is characterized in figure 9(e), which shows that the RIN spectrum is characterized by peaks at $f_{m}$ and 
the higher harmonics. The RIN spectrum is the worst among the investigated types; LF-RIN is $122 \mathrm{~dB} / \mathrm{GHz}$, which is $\sim 40 \mathrm{~dB} / \mathrm{Hz}$ higher than that of the non-modulated laser. The RIN spectrum of the PPPD type is plotted in figure 9(f), indicating that the spectrum is almost $30 \mathrm{~dB} / \mathrm{Hz}$ lower than that of the PPRO type but is higher than that of the non-modulated laser peaks. This spectrum is characterized by peaks at $f_{m} / 2, f_{m}$ and the higher harmonics. These results on the total RIN almost agree with those simulated by Ahmed for single mode AlGaAs lasers [13].

Dependence of the MC RIN in the low-frequency region, LF-RIN, on the modulation conditions is explored in figures 10. Figures 10(a)-(c) plot LF-RIN as a function of the modulation depth $m$ at frequencies $f_{m}=0.1 f_{r}, f_{m}=f_{r}$, and $f_{m}=1.4 f_{r}$, respectively. The figures plot RIN of both the total output and DM for both cases of nearly single-mode oscillation $\left(I_{b}=3 I_{t h}\right.$ with solid lines) and two-mode hopping oscillations $\left(I_{b}=2.74 I_{t h}\right.$ with dash lines). All figures indicate the modepartition effect that LF-RIN of DM is higher than that of the total output [33]. The figures indicate that the LF-RIN levels generally decreases little with the increase in the bias current from $I_{b}=2.74 I_{t h}$ to $I_{b}=3 I_{t h}$. This small decrease in LF-RIN seems to correspond to the improvement of the laser coherency associated with the increase in the injection current [33] rather than to the mode hopping effect. This result indicates that the sinusoidal modulation reduces the mode competition effect and suppresses the associated noise. The figures show that the total MC LF-RIN increases in general with the increase in $m$. The DM LF-RIN shows a similar behavior except that it decreases sharply with the increase in $m$ within $m<0.3$ when $f_{m}=f_{r}$ and $1.4 f_{r}$.

When $f_{m}=0.1 f_{r}$, figure 10(a) shows that LF-RIN of both the total output and DM increase with the increase in $m$. The total LF-RIN ranges between -141 and $-109 \mathrm{~dB} / \mathrm{Hz}$, while the DM LFRIN varies between -135 and $-92 \mathrm{~dB} / \mathrm{Hz}$. The LF-RIN levels are lowest under the CPS type, increase little when the modulated signal becomes CPSRO, and are highest for the PPRO type. That is, the noise performance and the laser coherence are worst in this regime of modulation. This result was also predicted in single-mode InGaAsP lasers [13] AlGaAs laser [15]. The DM LF-RIN saturates at $-92 \mathrm{~dB} / \mathrm{Hz}$ when $m>0.9$.

When $f_{m}=f_{r}$, figure $10(\mathrm{~b})$ shows that LF-RIN of the total output and DM of the nearly single-mode laser increase with the increase in $m$. In this case, LF-RIN is lowest within the 
operating range of the CPS type, increases within the operating range of the PP type, and highest under the PPPD type. The range of the total RIN is $(-164 \sim-145 \mathrm{~dB} / \mathrm{Hz})$ while it is $(-135 \sim-85$ $\mathrm{dB} / \mathrm{Hz}$ ) for the DM. On the other hand, the total RIN of the two-mode hopping case increases with the increase in $m$ within the operating range of the CPS type, and then decreases within the ranges of the PP and PPRD types. The DM LF-RIN decreases with the increase in $m$ when $m<0.3$ and then increases within the operating ranges of the CPS and PP types. Within the operating range of the PPRD type, this noise decreases with the increase in $m$. In this cases of $I_{b}=2.74 I_{t h}$, the range of the total RIN is $(-148 \sim-140 \mathrm{~dB} / \mathrm{Hz})$ while it is $(-128 \sim-145 \mathrm{~dB} / \mathrm{Hz})$ for the DM.

When $f_{m}=1.4 f_{r}$, figure $10(\mathrm{c})$ shows that LF-RIN of the total output and DM of the nearly single-mode oscillation increase with the increase in $m$ for the CPS types and then decreases within the operating ranges of the CPSPD and PPPD types. The range of the total RIN is $(-155 \sim-138$ $\mathrm{dB} / \mathrm{Hz})$ while it is $(-135 \sim-85 \mathrm{~dB} / \mathrm{Hz})$ for the DM. In the two-mode hopping case, the total and DM RIN levels decrease with the increase in $m$ up to $m=0.2$ and then increases within the relevant types of CPS, CPSPD and PPPD. In this case, the range of the total RIN is $(-150 \sim-137 \mathrm{~dB} / \mathrm{Hz})$ while it is $(-132 \sim-86 \mathrm{~dB} / \mathrm{Hz})$ for the $\mathrm{DM}$.

\subsection{Contribution of MC RIN to fiber link noise}

In this subsection, we use the simulated results of MC dynamics and RIN to evaluate the corresponding noise performance of RF fiber links in terms of NF.

Figures 11(a)-(c) illustrate variation of NF with the modulation depth $m$ at frequencies $f_{m}=$ $0.1 f_{r}, f_{m}=f_{r}$, and $f_{m}=1.4 f_{r}$, respectively for both cases of nearly single-mode oscillation $\left(I_{b}=3 I_{t h}\right.$ with solid lines) and two-mode hopping oscillations ( $I_{b}=2.74 I_{t h}$ with dash lines). The figure shows that NF generally increases with the increase in $m$ but decreases with the increase in $f_{m}$, which agrees with the experimental and theoretical findings by C. Carlsson and et al. [34] Therefore, NF attains lowest values within the operating conditions of the CPS type, whereas the operating conditions of the PPRO type result in the largest values. It is clear also that the CPS type corresponds to lower values of NF. On the other hand the high degree of non-uniformity of the PPRO and PPPD signals result in enhancement of NF. The figure indicates also that NF is larger 
when the laser exhibits two-mode hopping than when it supports nearly single mode oscillation. The difference between these NF values is clear for the low frequency $f_{m}=0.1 f_{r}$ as well as for $f_{m}=f_{r}$ when $m<0.7$.

Contribution of MC RIN to NF of the fiber link is quantified by comparing the calculated results of NF with those calculated by ignoring the laser RIN in Eq. (19). The latter results are also plotted in figure 11. The difference between both data then measures contribution of MC-RIN to NF. Figure 11 shows that the contribution of MC-RIN to NF increases with the decrease in $f_{m}$ except within the operating regions of the period doubling effect. This contribution increases with the increase in $m$ in general. As numerical illustrations, when $f_{m}=0.1 f_{r}$ the maximum contribution of MC RIN is $36 \mathrm{~dB}$ within the range of the CPS type and is $38 \mathrm{~dB}$ within the CPSRO region, whereas it is enhanced to $53 \mathrm{~dB}$ within the operating conditions of the PPRO type. When $f_{m}=f_{r}$ the maximum contribution of MC RIN is $22 \mathrm{~dB}$ within the ranges of both the CPS and PP types, which then decreases to $12 \mathrm{~dB}$ within the operating conditions of the PPPD type. When $f_{m}=1.4 f_{r}$ the maximum contribution of MC RIN reduces to $12 \mathrm{~dB}$ within the operating range of the CPS type, and reaches to $22 \mathrm{~dB}$ for signals with period doubling. On the other hand, when $f_{m}=1.4 f_{r}$, MC RIN has a negligible effect on the noise performance of the fiber link over a wide range of $m<1.0$ within which the laser exhibits the CPS type.

Figure 11 indicates also that NF due to the shot and thermal noises has lower values ranging between 30 37 dB. This means that the laser diode is the noisiest source in the link. The figure indicates also that modulating the laser diode up to $m=0.5$ with $f_{m}$ up to $f_{r}$ (which correspond to operation with the CPS type) results in smallest NF values (up to of $5 \mathrm{~dB}$ higher than the NF values without laser noise) as long as single mode oscillation is supported. The increase in NF due to the MC noise enhances when $f_{m}>f_{r}$ especially when laser emits PPRO and PPPD with higher degree of non-uniformity.

\section{Conclusions}

We introduced comprehensive large-signal analyses of longitudinal MC and the associated noise in semiconductor lasers under sinusoidal modulation. Contribution of MC noise to NF of directly modulated short fiber links is quantified. The MC RIN properties are correlated to the time-domain 
characteristics of the signals considering cases of nearly single-mode oscillation and two-mode hopping.

The modulated laser waveform is classified into three types of continuous variation with time; namely CPS, CPSRO and CPSPD, and three pulsing types; namely, PP, PPRO and PPPD. The classification is independent of the modal oscillation of the laser. The CPS type dominates the range of low values of $m$. When $f_{m}$ is low, the increase in $m$ converts the CPS type into the CPSRO type and then to the PPRO type. When $f_{m}$ is around $f_{r}$, the laser output is uniform, either the CPS type or PP type. When $f_{m} / f_{m}$ is large with $m>1.2$, the CPS and PPPD types dominates. The laser keeps oscillation in single mode when the signal is continuous, whereas the pulsing signals are characterized by multimode oscillation.

In the non-modulated laser, the total $\mathrm{RIN}$ is $15 \mathrm{~dB} / \mathrm{Hz}$ higher under two mode-hopping than under nearly single-mode oscillation. This difference increases to $70 \mathrm{~dB} / \mathrm{Hz}$ for the $\mathrm{DM} \mathrm{RIN} / \mathrm{Hz}$. Under modulation, mode hopping is stabilized and the RIN differences are reduced to the effect of injection current. The total MC LF-RIN increases in general with the increase in $m$. The DM LFRIN shows a similar behavior except that it decreases sharply with the increase in $m$ within $m<0.3$ when $f_{m}=f_{r}$ and $1.4 f_{r}$. The LF-RIN levels are highest under the PPRO type when $f_{m}=0.1 f_{r}$, and lowest under the CPS type when $f_{m}=1.4 f_{r}$. When $f_{m} / f_{r}$ is low, LF-RIN is lowest under the CPS type, increases little when the modulated signal becomes CPSRO, and is highest for the PPRO type. When $f_{m} / f_{r}$ is large, LF-RIN is lowest under the CPS type, increases within the range of the PP type, and highest under the PPPD type.

NF of the directly modulated fiber link generally increases with the increase in $m$ but decreases with the increase in $f_{m}$. NF attains smallest values under the CPS type, and largest values under the PPRO type. The contribution of MC noise to NF increases with the decrease in $f_{m}$ except within the regimes of period doubling. This contribution increases with the increase in $m$ in general. When $f_{m}=0.1 f_{r}$, the maximum contribution of RIN is $36 \mathrm{~dB}$ within the range of the CPS type, and is enhanced to $53 \mathrm{~dB}$ under the PPRO type. When $f_{m}=f_{r}$ the contribution of RIN reaches $22 \mathrm{~dB}$ within the ranges of the CPS and PP types, which decreases to $12 \mathrm{~dB}$ under the PPPD type. When $f_{m}=1.4 f_{r}$, 
RIN has negligible influence on $N F$ over a wide range of $m<1.0$ within which the laser exhibits the CPS type.

\section{Acknowledgment}

This paper was funded by the Deanship of Scientific Research (DSR), King Abdulaziz University, Jeddah, Saudi Arabia, under grant No. (16-130-D1432). The authors, therefore, acknowledge with thanks DSR technical and financial support.

\section{References}

[1] Cox C 2004 Analog Optical Links Theory and Practice (Cambridge University Press).

[2] Cox C and Chang W S 2002 RF Photonic Technology in Optical Fiber Links (Cambridge University Press).

[3] Larsson A, Carlsson C, Gustavsson J, Haglund A, Modh P and Bengtsson J 2004 New .J. Phys 61.

[4] Yamada M 1986 IEEE J Quantum Electron QE-22 1052.

[5] Ahmed M and Yamada M 2002 IEEE J Quantum Electron 38682.

[6] Ahmed M 2003 Physics D 176212.

[7] Yamada M, Ishimori W, Sakaguchi H Ahmed M 2003 IEEE J Quantum Electron 391548.

[8] Lau K Y, Gee C M, Chen T R, Bar-Chaim N and Ury I 2993 J. Lightwave Technol. 11 1216.

[9] Lau K Y and Blauvelt H 2988 Appl. Phys. Lett. 5269.

[10] Chusseau L, Henery E and Lourtioz J M 1989 Appl Phys Lett. 55822.

[11] Henery E, Chusseau L and Lourtioz J M 1990 IEEE J Quantum Electron. 26633.

[12] Ahmed M and El-Lafi A 2008 Opt. Laser Technol. 40809.

[13] Ahmed M 2008 J. Phys. D 41 175104-1.

[14] Azadeh M 2009 Fiber Optics Engineering (New York: Springer).

[15] Ahmed M, El-sayed N Z and Ibrahim H 2012 Europ. J. Phys. D, Accepted.

[16] Osinski M and Buus J 2987 IEEE J. Quantum Electron. QE-23 9.

[17] Ahmed M, Yamada M and Saito M 2001 IEEE J. Quantum Electron. 371600.

[18] Marcuse D 2984 IEEE J. Quantum Electron. QE-20 1139

[19] Stuart A and Ord J K 1980 Advanced Theory of Statistics (London: Griffin).

[20] Yamada M 1989 J. Appl. Phys. 6681.

[21] Ogasawara N and Ito R 1988 Jpn. J. Appl. Phys. 27607.

[22] Ohtsu M, Otsuka Y, and Teramachi Y 1989 IEEE J. Quantum Electron. 2531. 
[23] Yamada M 1994 IEEE J. Quantum Electron. 301511.

[24] Ahmed M, Yamada M and Abdulrhmann S 2001 Fluct. Noise Lett. 1, L163.

[25] Henry C H, Henry P S and Lax M 2984 J. Lightwave Technol. LT-2 209.

[26] Abbas G L and Yee T K 1985 IEEE J. Quantum Electron. QE-21, 1303.

[27] Gray G and Roy R 1989 Phys. Rev. A 402453.

[28] Lee C H, Yoon T H and Shin S Y 1984 Appl Phys Lett. 4695.

[29] Ahmed M and El-Lafi A 2008 Pramana J. Phys. 7190.

[30] Agrawal G P and Dutta N K 1993 Semiconductor Lasers (New York: Van Nostrand Reinhold).

[31] Kao Y H and Lin H T 1993 Phys. Rev. A 482292.

[32] Lau K Y, Harder C and Yariv A 1984 IEEE J. Quantum Electron. QE-20 71

[33] Anderson T B and Clarke B R 1993 IEEE J. Quant. Electron. 293.

[34] Carlsson C Larsson A and Alping A 2004 J. Lightwav. Technol. 221694. 

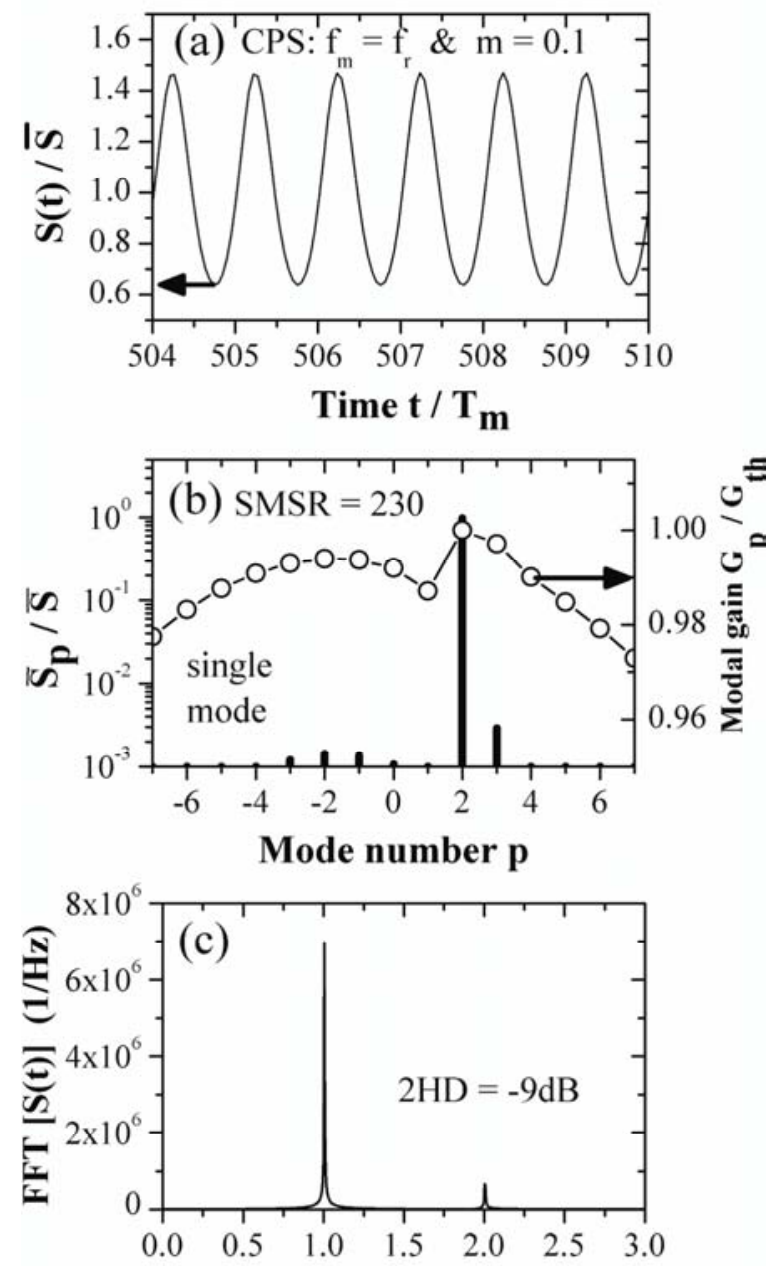

Fourier frequency $f / \mathbf{f}_{\mathbf{m}}$

Figure 1. Simulation characteristics of the non-modulated laser diode: (a) and (b) time variation of $S_{p}(t)$ of the strongest modes, (c) and (d) modal gain and intensity spectrum, (d) and (e) RIN spectra of the total output and strongest modes, when $I=2.76$ and $3.0 I_{t h}$, respectively. 


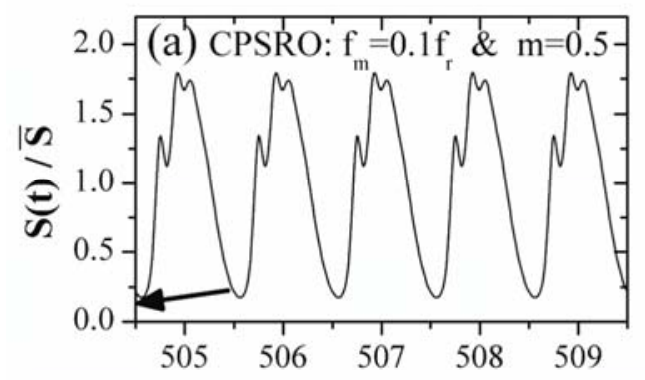

Time $\mathbf{t} / \mathbf{T}_{\mathbf{m}}$

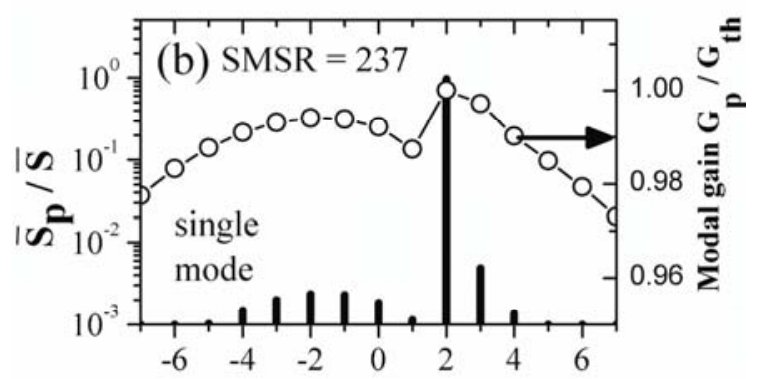

Mode number $\mathbf{p}$

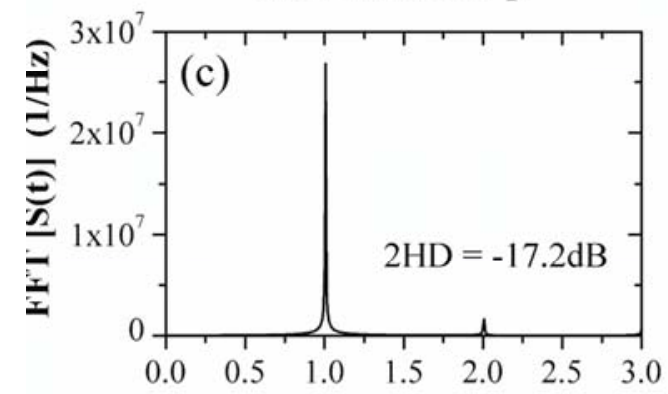

Fourier frequency $f / f_{m}$

Figure 2. Characteristics of the CPS modulation type: (a) waveform of $S(t)$, (b) spectra of mode intensities and gain, and (3) FFT frequency spectrum $S_{f}$, when $f_{m}=f_{r}$ and $m=0.1$. The signal varies continuously and periodical with time and the laser output is single mode. 

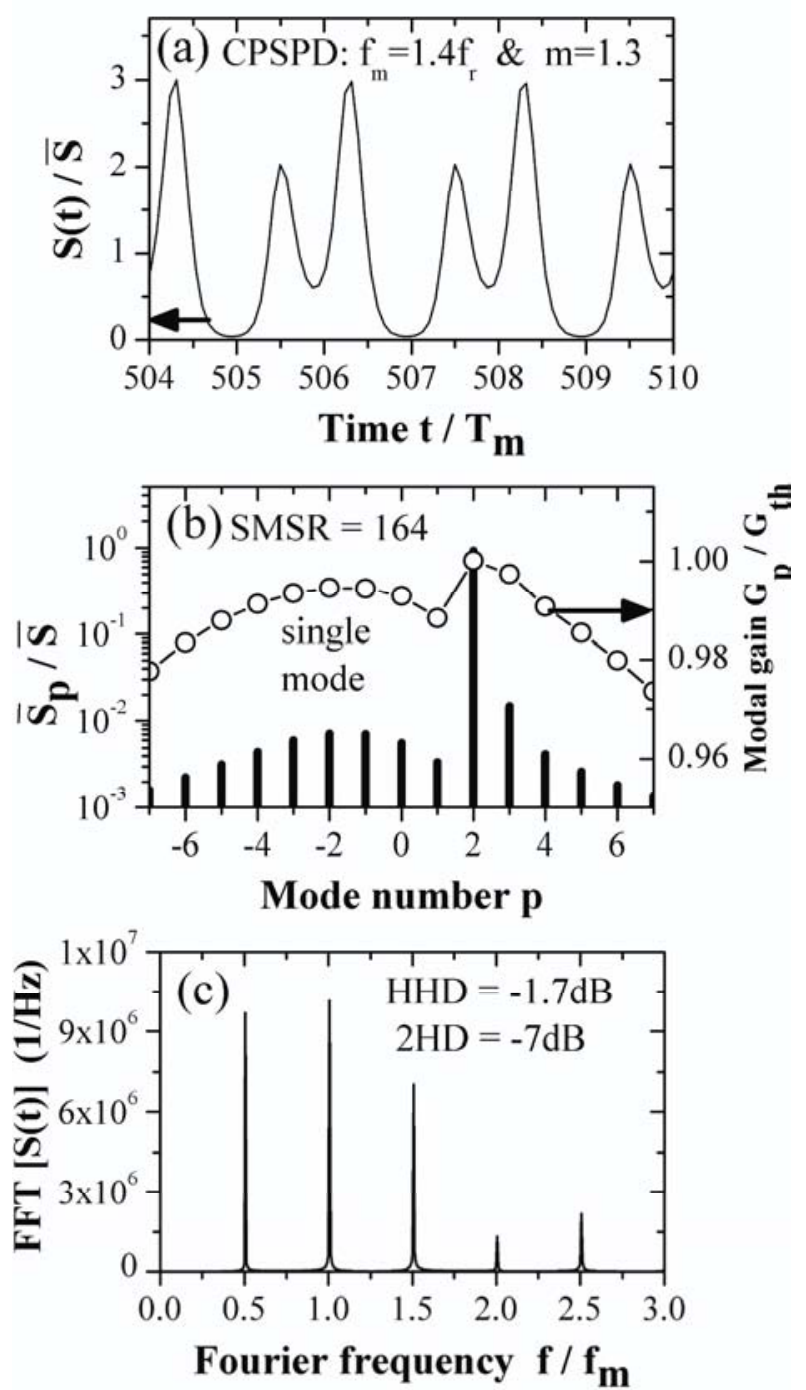

Figure 3. Characteristics of the CPSRO modulation type: (a) waveform of $S(t)$, (b) spectra of mode intensities and gain, and (3) FFT frequency spectrum $S_{f}$, when $f_{m}=0.1 f_{r}$ and $m=0.5$. The laser output is single mode. 

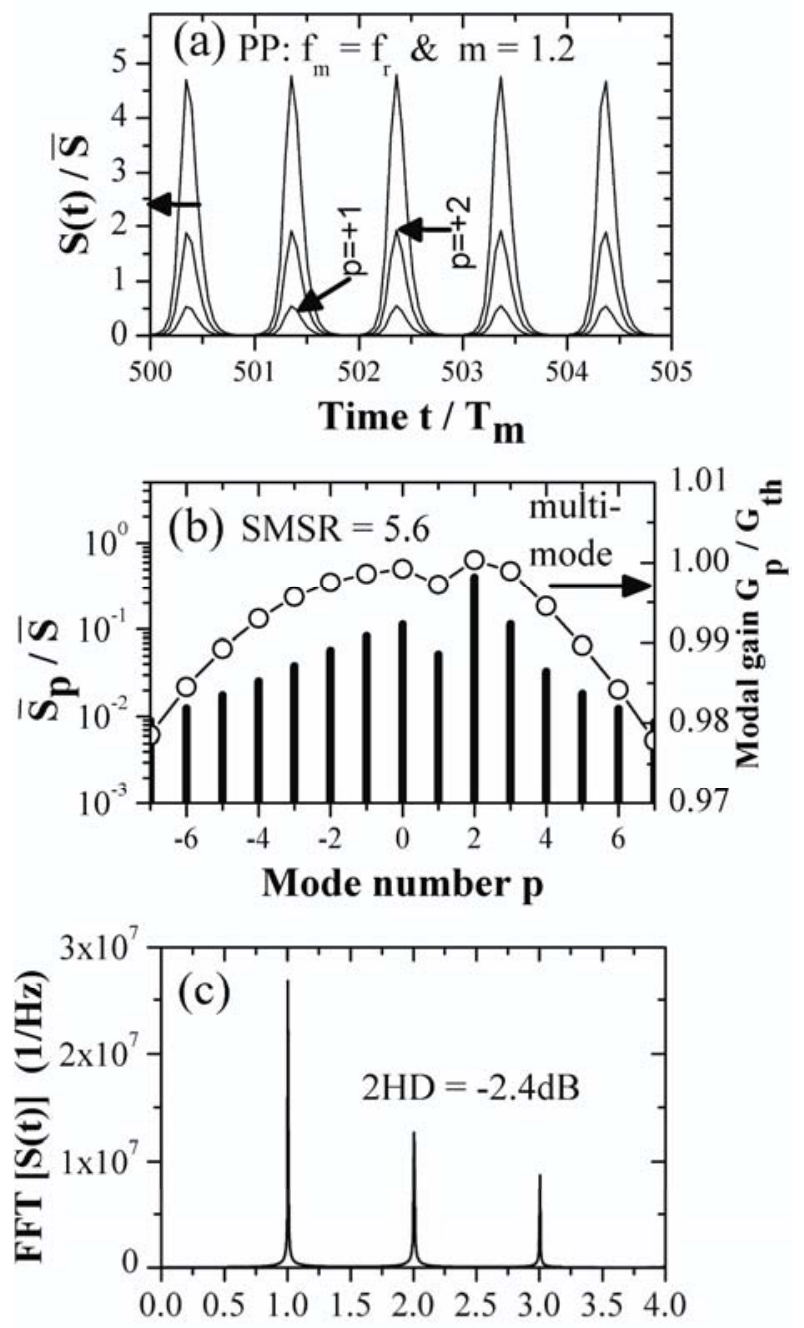

Fourier frequency $\mathbf{f} / \mathbf{f}_{\mathbf{m}}$

Figure 4. Characteristics of the CPSPD modulation type: (a) waveform of $S(t)$, (b) spectra of mode intensities and gain, and (3) FFT frequency spectrum $S_{f}$, when $f_{m}=f_{r}$ and $m=1.3$. The signal had period doubling and the laser output is single mode. 

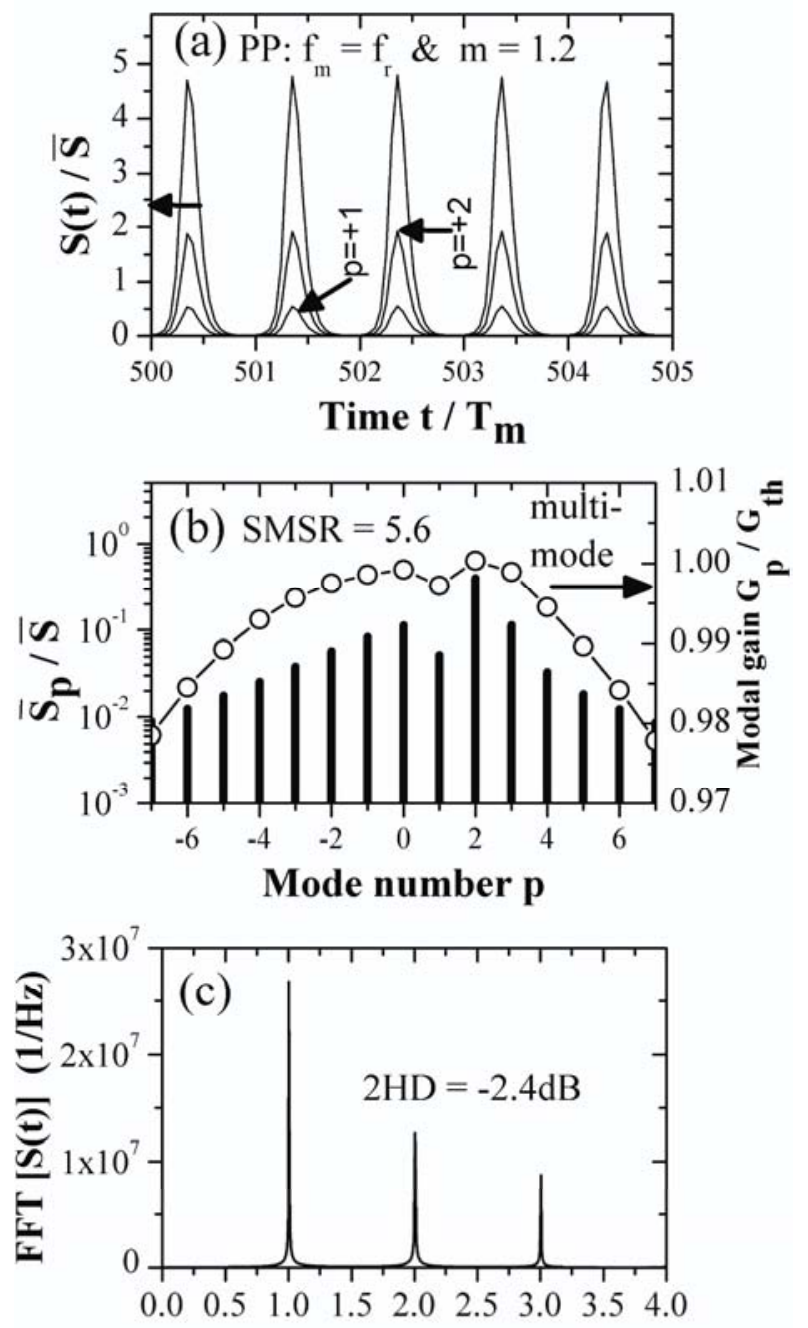

Fourier frequency $\mathbf{f} / \mathbf{f}_{\mathbf{m}}$

Figure 5. Characteristics of the PP modulation type: (a) waveform of $S(t)$, (b) spectra of mode intensities and gain, and (3) FFT frequency spectrum $S_{f}$, when $f_{m}=f_{r}$ and $m=1$.2. The laser emits periodic pulses and its output is single mode. 

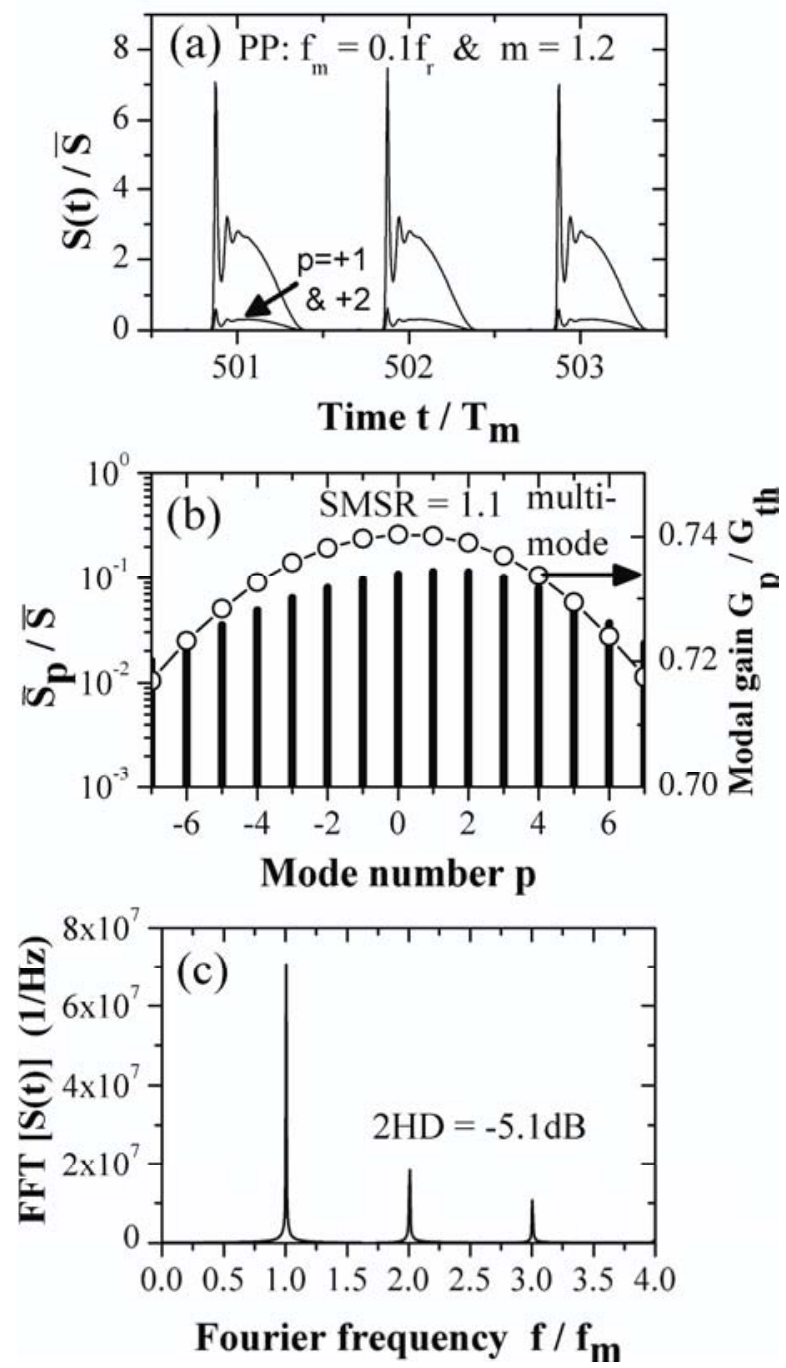

Figure 6. Characteristics of the PPRO modulation type: (a) waveform of $S(t)$, (b) spectra of mode intensities and gain, and (3) FFT frequency spectrum $S_{f}$, when $f_{m}=0.1 f_{r}$ and $m=1.2$. The pulses are superposed by relaxation oscillations and the laser output is single mode. 

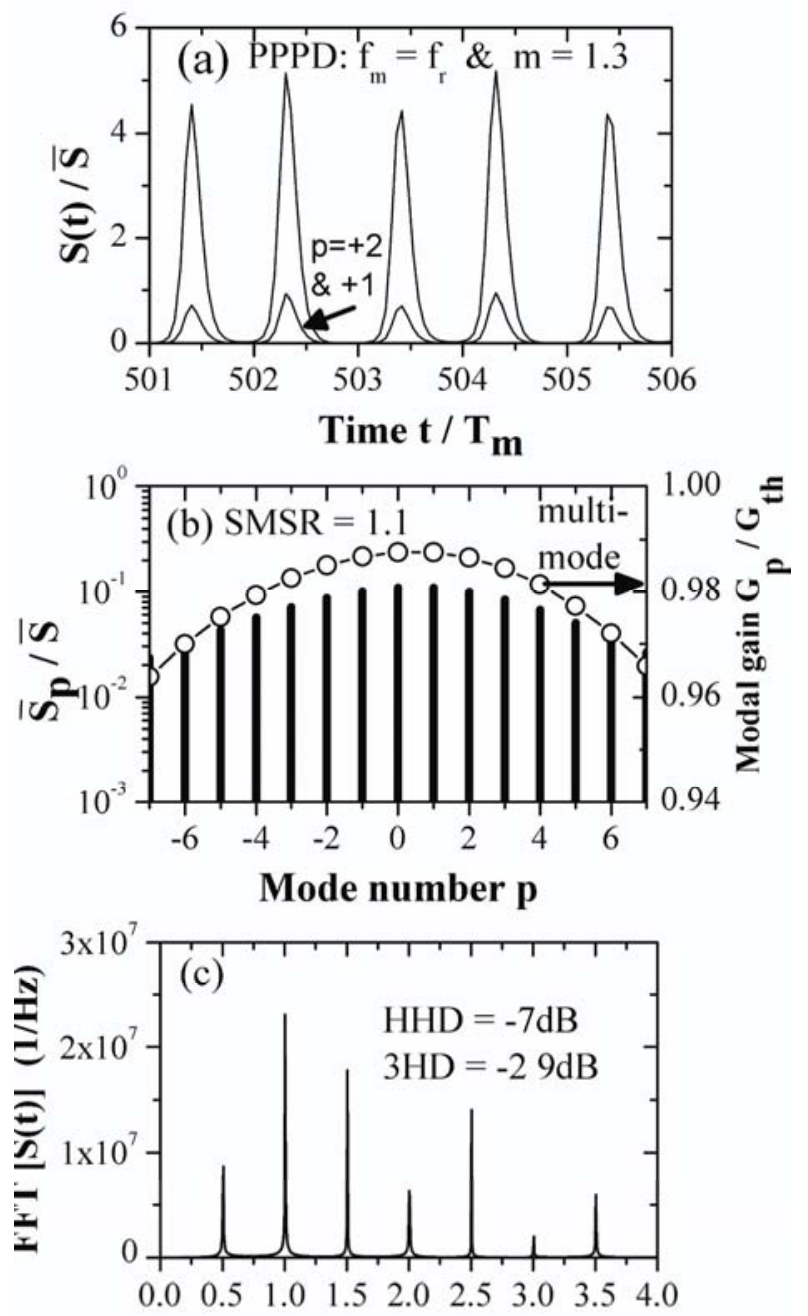

Fourier frequency $f / \mathbf{f}_{\mathbf{m}}$

Figure 7. Characteristics of the PPPD modulation type: (a) waveform of $S(t)$, (b) spectra of mode intensities and gain, and (3) FFT frequency spectrum $S_{f}$, when $f_{m}=f_{r}$ and $m=1.3$. The signal had period doubling and the laser output is multimode. 


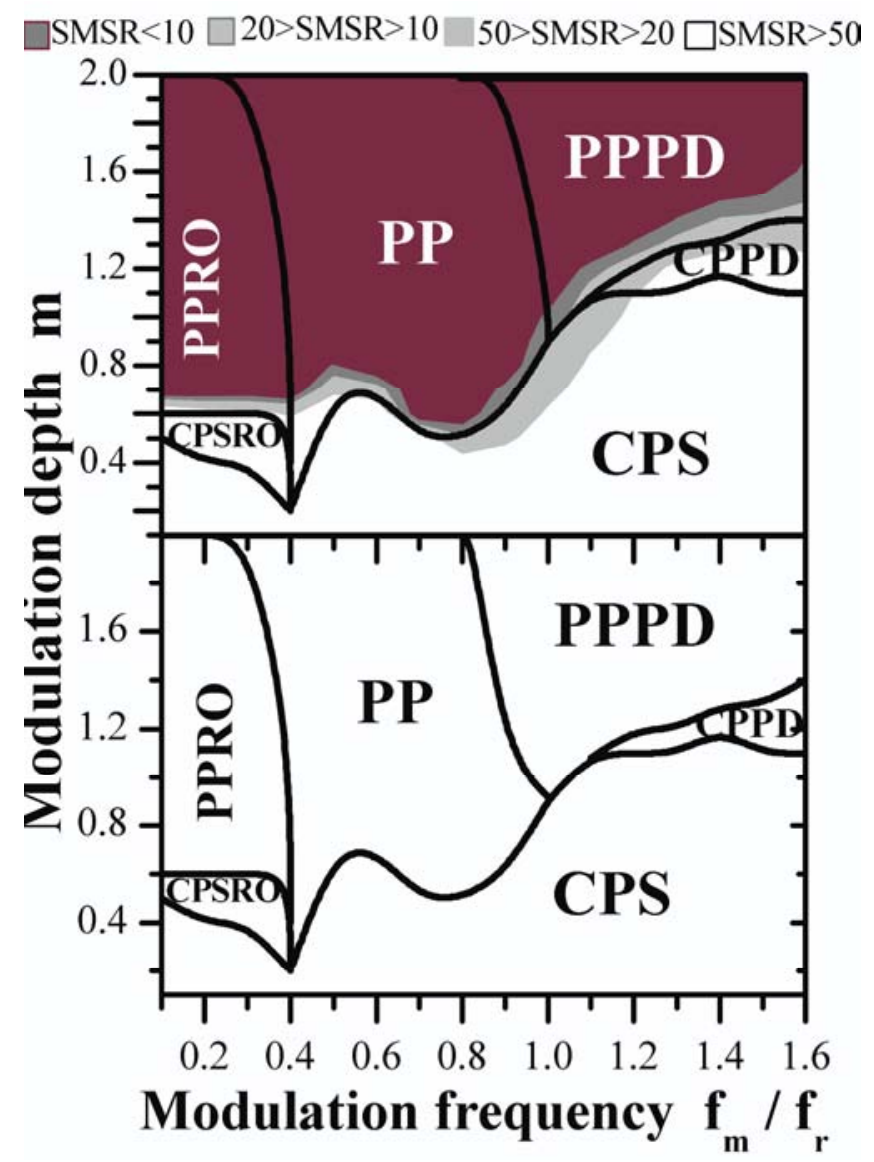

Figure 8. ( $m$ versus $f_{m} / f_{r}$ ) diagram of the types of the modulated laser signal when $I_{b}=3 I_{t h}$ for: (a) the present nearly single-mode laser, and (b) single mode laser. In (a), a contour plot of SMSR is included. 

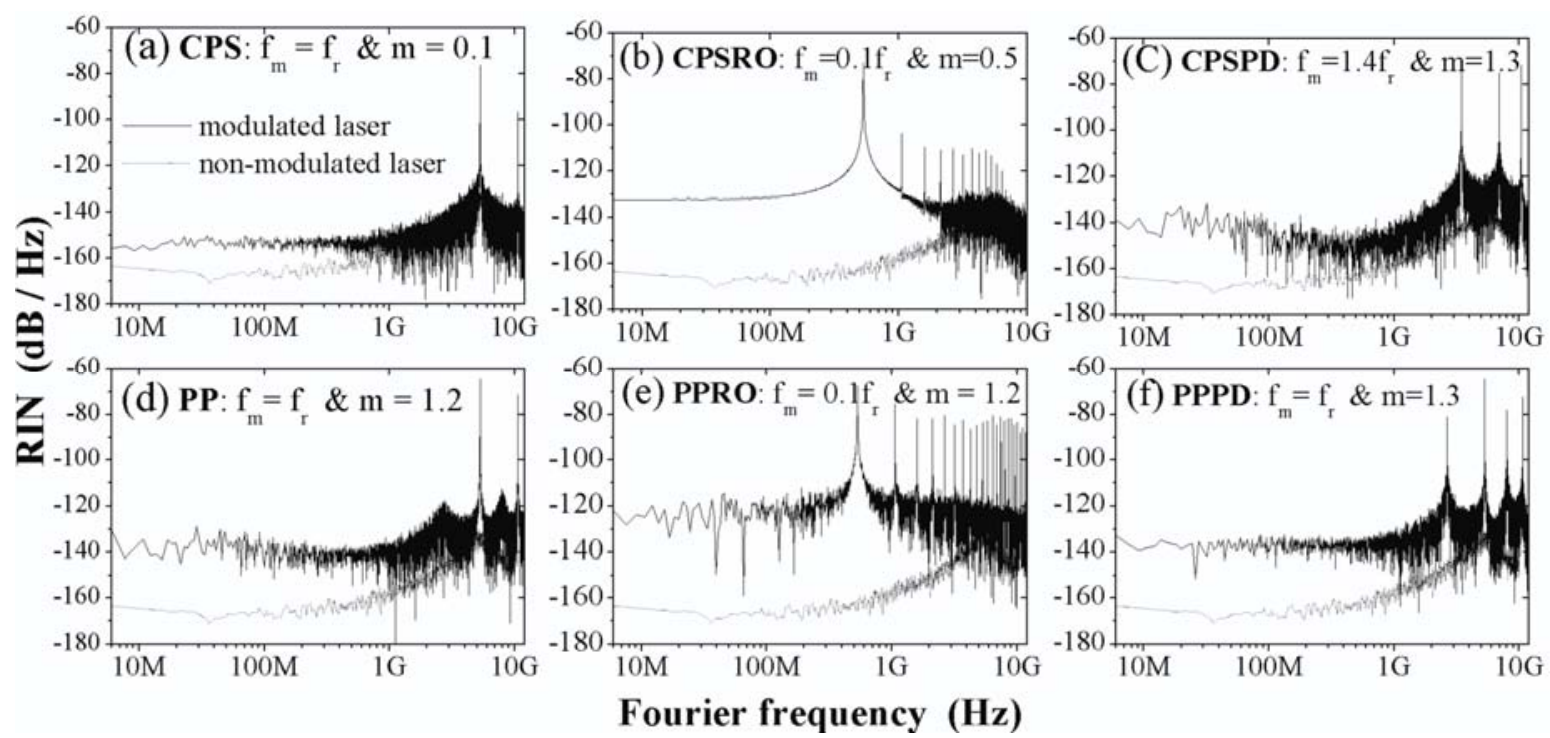

Figure 9. Frequency spectrum of RIN (a) CPS ( $\left.m=0.1 \& f_{m}=f_{r}\right)$, (b) CPSRO ( $m=0.1 \& f_{m}=0.5 f_{r}$ ), (c) CPSPD $\left(m=1.3 \& f_{m}=1.4 f_{r}\right)$, (d) PP $\left(m=1.2 \& f_{m}=f_{r}\right)$, (e) PPRO $\left(m=1.1 \& f_{m}=\right.$ $\left.0.1 f_{r}\right)$, and (g) PPPD $\left(m=1.3 \& f_{m}=f_{r}\right)$. The RIN spectrum of the non-modulated laser is also plotted by dashed lines for comparison. 


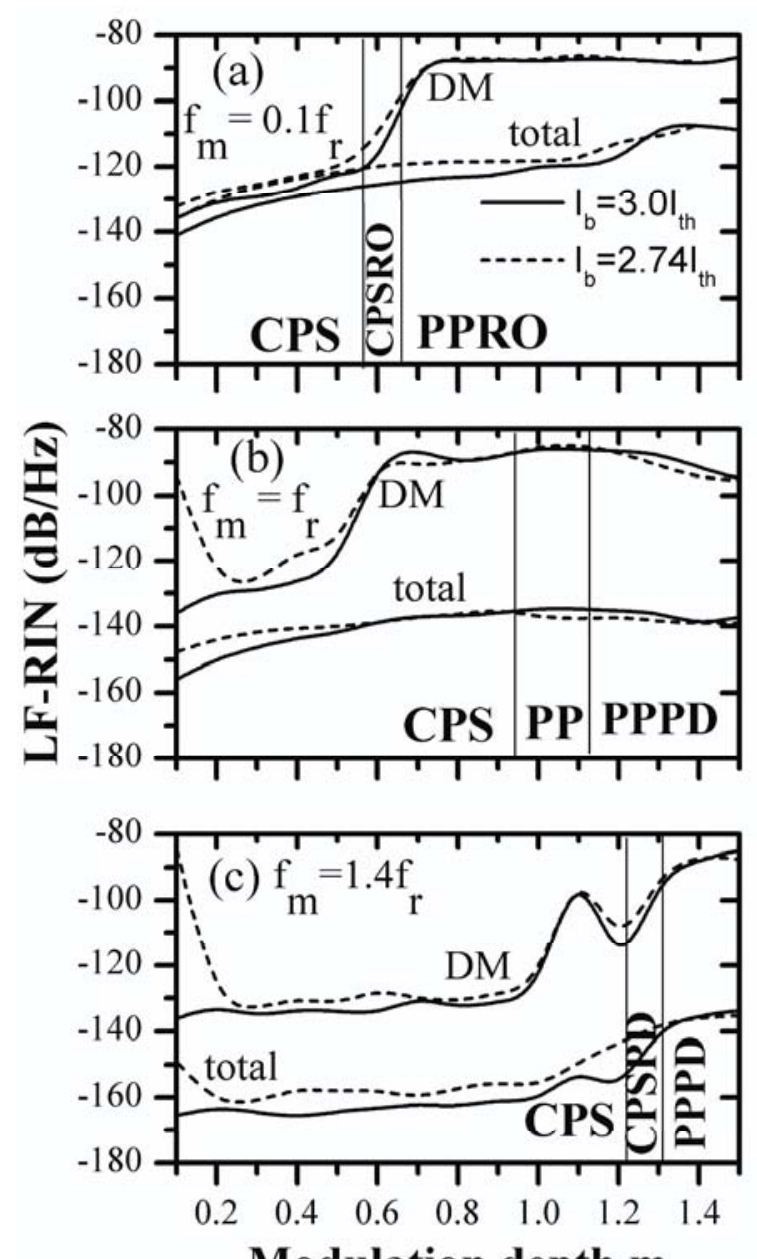

Modulation depth $\mathbf{m}$

Figure 10. Variations of RIN of the total output and DM with modulation depth $m$ when (a) $f_{m}=0.1 f_{r}$, (b) $f_{m}=f_{r}$, and (c) $f_{m}=1.4 f_{r}$. The type of the modulated signal is written within the corresponding operating ranges of $m$. 

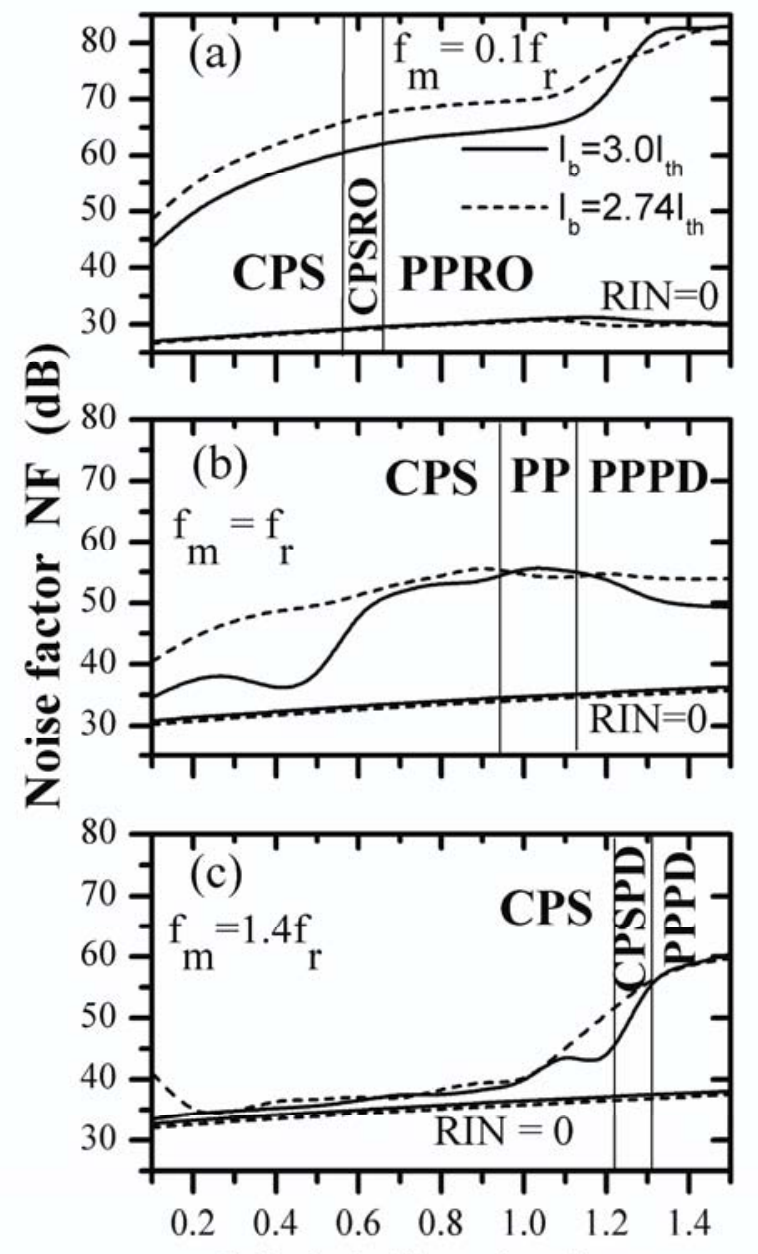

Modulation depth $\mathbf{m}$

Figure 11. Variation of $N F$ of the fiber link with $m$ when (a) $f_{m}=0.1 f_{r}$, (b) $f_{m}=f_{r}$, and (c) $f_{m}=1.4 f_{r}$. The type of the modulated signal is written within the corresponding operating ranges of $m$. The results when MC RIN $=0$ are also shown. 
Table 1. Definitions and values of the parameters of the AlGaAs laser and fiber link used in the present calculations.

\begin{tabular}{|c|c|c|c|}
\hline Symbol & Meaning & Value & Unit \\
\hline \multicolumn{4}{|c|}{$\underline{\text { Laser parameters }}$} \\
\hline$\lambda$ & Emission wavelength & 850 & $\mathrm{~nm}$ \\
\hline$a$ & Tangential gain coefficient & $2.75 \times 10^{-12}$ & $\mathrm{~m}^{3} \mathrm{~s}^{-1}$ \\
\hline$\xi$ & Field confinement factor in the active layer & 0.2 & --- \\
\hline$b$ & $\begin{array}{c}\text { dispersion parameter of the linear gain } \\
\text { spectrum }\end{array}$ & $3 \times 10^{19}$ & $m^{3} A^{-2}$ \\
\hline$\alpha$ & Linewidth enhancement factor & 2.6 & -- \\
\hline$V$ & Volume of the active region & $150 \times 10^{-18}$ & $\mathrm{~m}^{3}$ \\
\hline$N_{g}$ & Electron number at transparency & $2.1 \times 10^{8}$ & --- \\
\hline C & Spontaneous emission factor & $1.023 \times 10^{-5}$ & --- \\
\hline$\delta \lambda$ & half-width of spontaneous emission & 23 & $\mathrm{~nm}$ \\
\hline$\tau_{\mathrm{s}}$ & Spontaneous emission lifetime & $2.79 \times 10^{-9}$ & s \\
\hline$\left|R_{c v}\right|^{2}$ & Squared absolute value of the dipole moment & $2.8 \times 10^{-57}$ & $\mathrm{C}^{2} \mathrm{~m}^{2}$ \\
\hline$\tau_{i n}$ & electron intraband relaxation time & 0.1 & ps \\
\hline$N_{s}$ & $\begin{array}{c}\text { Electron number characterizing gain } \\
\text { suppression }\end{array}$ & $1.7 \times 10^{8}$ & --- \\
\hline$L_{D}$ & Length of the active region & 300 & $\mu \mathrm{m}$ \\
\hline$n_{D}$ & Refractive index of the active region & 3.6 & - \\
\hline$R_{f}$ & Reflectivity of front facet & 0.3 & -- \\
\hline$R_{b}$ & Reflectivity of back facet & 0.6 & -- \\
\hline$G_{t h}$ & Threshold gain level & $3.22 \times 10^{11}$ & $\mathrm{~s}^{-1}$ \\
\hline$I_{\text {th }}$ & Threshold current & 15.4 & $\mathrm{~mA}$ \\
\hline \multicolumn{4}{|c|}{$\underline{\text { Fiber link parameters }}$} \\
\hline$\alpha_{\text {loss }}$ & Fiber loss & -0.2 & $\mathrm{~dB}$ \\
\hline$R$ & Responsivity of photodiode & 0.6 & $\mathrm{~mA} / \mathrm{mW}$ \\
\hline$I_{d}$ & Dark current of photodiode & 0.2 & $\mathrm{~mA}$ \\
\hline$R_{S}$ & Series diode resistance & 10.6 & $\Omega$ \\
\hline$C_{d}$ & Junction capacitance & 170 & $\mathrm{pF}$ \\
\hline$R_{\text {match }}$ & Matching resistance & 50 & $\Omega$ \\
\hline$R_{L}$ & Load resistance & 50 & $\Omega$ \\
\hline$N_{d}$ & Number of transformer twist & 2 & -- \\
\hline
\end{tabular}

